\title{
Representing Range Compensators with Computational Geometry in TOPAS
}

\author{
Forrest N. Iandola \\ University of Illinois at Urbana-Champaign \\ and SLAC National Accelerator Laboratory, Menlo Park, CA 94025
}

August 19, 2011

Prepared in partial fulfillment of the requirements of the Department of Energy's Science Undergraduate Laboratory Internship under the direction of Joseph Perl.

Participant:

Signature

Research Advisor:

Signature 


\section{TABLE OF CONTENTS}

Abstract

$\begin{array}{ll}\text { Introduction } & 1\end{array}$

$\begin{array}{ll}\text { Background } & 1\end{array}$

Range Compensator Model with Subtraction Solids 3

"3D Hexagon"-based Range Compensator Approximation 3

Conclusions and Future Work 4

$\begin{array}{ll}\text { Acknowledgments } & 4\end{array}$

$\begin{array}{ll}\text { References } & 4\end{array}$ 


\begin{abstract}
In a proton therapy beamline, the range compensator modulates the beam energy, which subsequently controls the depth at which protons deposit energy. In this paper, we introduce two computational representations of range compensator. One of our compensator representations, which we refer to as a subtraction solid-based range compensator, precisely represents the compensator. Our other representation, the 3D hexagon-based range compensator, closely approximates the compensator geometry. We have implemented both of these compensator models in a proton therapy Monte Carlo simulation called TOPAS (Tool for Particle Simulation). In the future, we will present a detailed study of the accuracy and runtime performance trade-offs between our two range compensator representations.
\end{abstract}




\section{INTRODUCTION}

Proton therapy is one of the best methods available for treating cancer [1]. The number of proton facilities is steadily increasing, and proton therapy is entering the mainstream of radiation therapy. TOPAS (Tool for Particle Simulation) is a four year NIH-funded project of SLAC, University of California, San Francisco (UCSF) and Massachusetts General Hospital to make Monte Carlo particle transport simulation faster and easier to use for proton therapy [2] [3] [4]. TOPAS enables researchers and clinicians to refine proton therapy delivery system designs and individual patient treatment plans to improve proton therapy's therapeutic ratio (improved tumor control, reduced side effects). TOPAS offers a collection of ready-made geometries for modeling proton therapy beamlines, and TOPAS uses Geant4 for fundamental physics processes.

In most proton therapy facilities, an extensive beamline lies between the proton source and the patient. This beamline includes components such as collimators, magnets, a range modulator, scatterers, and a range compensator. The range compensator (or simply compensator) sits close to the patient. The fundamental purpose of a range compensator is to modify the proton beam energy so that the protons deliver energy to the correct location in a patient. This study seeks to represent compensators with computational geometry. While a wide variety of computational geometry methods could be applied to compensator representation, we focus on fast and accurate methods. This paper presents two compensator representations, which the authors have recently implemented in TOPAS.

\section{BACKGROUND}

\section{The Range Compensator}

In proton treatment facilities, medical staff typically prescribe a radiation dose (in Greys) to a patient's tumor. To ensure that the correct dose is delivered to the correct location, the 
protons must have a specific energy. The necessary energy may vary within the treatment region. A range compensator allows a beamline to deliver protons with the correct energy (and corresponding depth of deposition).

In many proton therapy facilities, a non-Monte Carlo treatment planning software such as $\mathrm{XiO}$ [5] designs a unique range compensator for each patient. In some facilities, medical physicists use a Monte Carlo simulation such as TOPAS to validate the compensator design. Therapy facilities typically construct compensators by using a milling machine to drill a number of holes out of a cylinder of Lucite or other clear plastic. Each drill hole may have a unique depth, such as in Figure 1. The thickness of the Lucite proportionally reduces the protons' energy. Lower-energy protons deposit most of their energy at a shorter depth in the patient than higher-energy protons. In short, the range compensator modulates the beam energy, which subsequently controls the depth at which protons deposit energy.

\section{Subtraction Solids in Geant4}

Geant4 supports boolean solid combinatorial geometry [6]. Subtraction solids are a type of Geant4 boolean solid. Subtraction solids enable a small solid to be cut out, or subtracted, from a larger solid [7]. The pseudo code in Figure 2 serves as a simple example of subtraction solid geometry. As shown in Figure 3, Geant4 also allows nested subtraction of solids. In addition, subtracted solids are allowed to overlap. Therefore, for the example in Figure 3, Geant4's ability to track particles is not impaired if the subtracted cylinders, smallCylinder1 and smallCylinder2, overlap. In contrast, geometrical components that are not comprised of boolean solids are not allowed to overlap in Geant4. 


\section{RANGE COMPENSATOR MODEL WITH SUBTRACTION SOLIDS}

Given the compensator milling machine instructions in .Decimal format [8], we subtract each drill hole from the solid Lucite cylinder. We subtract the cylinders in the same order that the milling machine would drill the cylinders. Thus, we produce an exact model of the real compensator. The pseudo code in Figure 3 constructs a compensator comprised of a bigCylinder with $\mathrm{n}$ drill holes subtracted. Figure 4 offers a Geant4 visualization [9] of a subtraction solid compensator with twelve holes subtracted.

Our (unpublished) preliminary results show that, especially for compensators with fifty or more drill holes, tracking particles through a subtraction solid-based compensator is quite slow. To remedy this, we present a hexagon-based compensator approximation in the next section.

\section{"3D HEXAGON"-BASED RANGE COMPENSATOR APPROXIMATION}

In this section, we present an approximate compensator representation. We approximate the drill holes with a collection of "3D hexagons," as shown in Figure 5. Each 3D hexagon has the depth of the drill hole that it represents.

We choose 3D hexagons, because they can be clustered without overlap or gaps. (Recall that, while boolean solids allow overlap, other forms of Geant4 geometry do not allow

overlap.) The lack of overlap among the hexagons allows us to insert a volume of air in the shape of each 3D hexagon into the Lucite cylinder. However, unlike the precise compensator model produced with subtraction solids, the 3D hexagon model does not correctly represent the drill hole depth in regions where drill holes overlap. 
Our preliminary results show that particle tracking through a hexagon-based compensator is as up to twenty times faster than through a subtraction solid-based compensator. We will present the finalized version of these results in a future study.

\section{CONCLUSIONS AND FUTURE WORK}

In this paper, we introduced two range compensator representations, which we have implemented in TOPAS. In the near future, we will present a detailed study of the accuracy and runtime performance trade-offs between our two compensator representations.

\section{ACKNOWLEDGMENTS}

The authors thank Jan Schuemann, Harald Paganetti, Jungwook Shin, and Bruce Faddegon for their helpful suggestions. Thanks to the U.S. Department of Energy and the National Institutes of Health for their generous support.

\section{REFERENCES}

[1] H. Suit, S. Goldberg, A. Niemierko, A. Trofimov, J. Adams, H. Paganetti, G. Chen, T. Bortfeld, S. Rosenthal, J. Loeffler, and T. Delaney, "Proton beams to replace photon beams in radical dose treatments." Acta Oncologica, vol. 42, no. 8, pp. 800-808, 2003.

[2] J. Perl, J. Shin, J. Schuemann, B. Faddegon, and H. Paganetti, "Topas: A fast and easy to use tool for particle simulation," in Joint American Association of Physicists in Medicine (AAPM) and Canadian Organization of Medical Physicists (COMP) Meeting, August 2011. 
[3] J. Perl, J. Schuemann, J. Shin, B. Faddegon, and H. Paganetti, "Topas: A fast and easy to use monte carlo system for proton therapy," in Presented at the 50th Meeting of the Particle Therapy Co-Operative Group (PTCOG), May 2011.

[4] T. Akagi, T. Aso, B. Faddegon, A. Kimura, N. Matsufuji, T. Nishi, C. Omachi, H. Paganetti, J. Perl, T. Sasaki, D. Sawkey, J. Scheumann, J. Shin, T. Toshito, T. Yamashita, and Y. Yoshida, "The ptsim and topas projects, bringing geant4 to the particle therapy clinic," in Joint International Conference on Supercomputing in Nuclear Applications and Monte Carlo 2010 (SNA + MC 2010), October 2010.

[5] M. Lee, "Xio treatment planning system from elekta cms software optimizes clinical treatment at rinecker proton therapy center," Elekta, Inc. Press Release, October 2009, http://www.elekta.com/healthcare_international_press_release_20070632.php.

[6] G. Cosmo, "Modeling detector geometries in geant4," in IEEE Nuclear Science Symposium (IEEE-NSS), vol. 1, October 2003, pp. 479-481.

[7] — , "The geant4 geometry modeler," in IEEE Nuclear Science Symposium (IEEENSS), vol. 4, October 2004, pp. 2196-2198.

[8] B. E. Willis, "Commissioning of adac pinnacle for imrt utilizing solid compensators," Master's thesis, Oregon State University, 2008.

[9] J. Allison, M. Asai, G. Barrand, M. Dönszelmann, K. Minamimoto, J. Perl, S. Tanaka, E. Tcherniaev, and J. Tinslay, "The geant4 visualisation system," Computer Physics Communications, vol. 178, no. 5, pp. 331-365, 2008. 


\section{FIGURES}

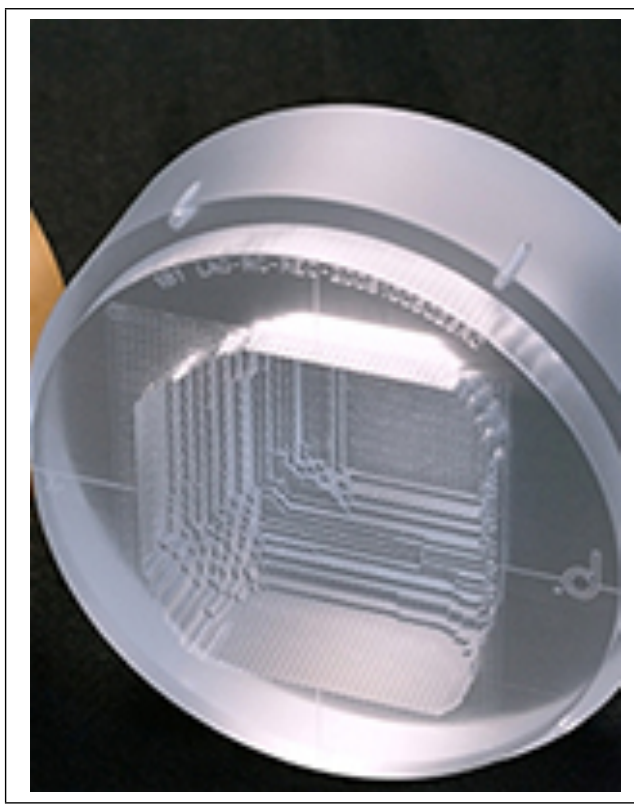

Figure 1: Example range compensator

newSolid = bigCylinder - smallCylinder

Figure 2: Pseudo-code for simple subtraction solid example

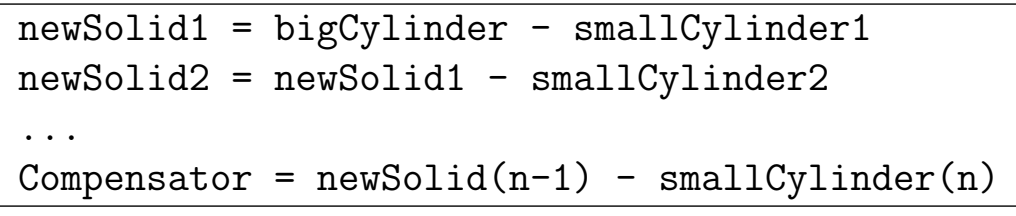

Figure 3: Pseudo-code for repeated subtraction solid example 


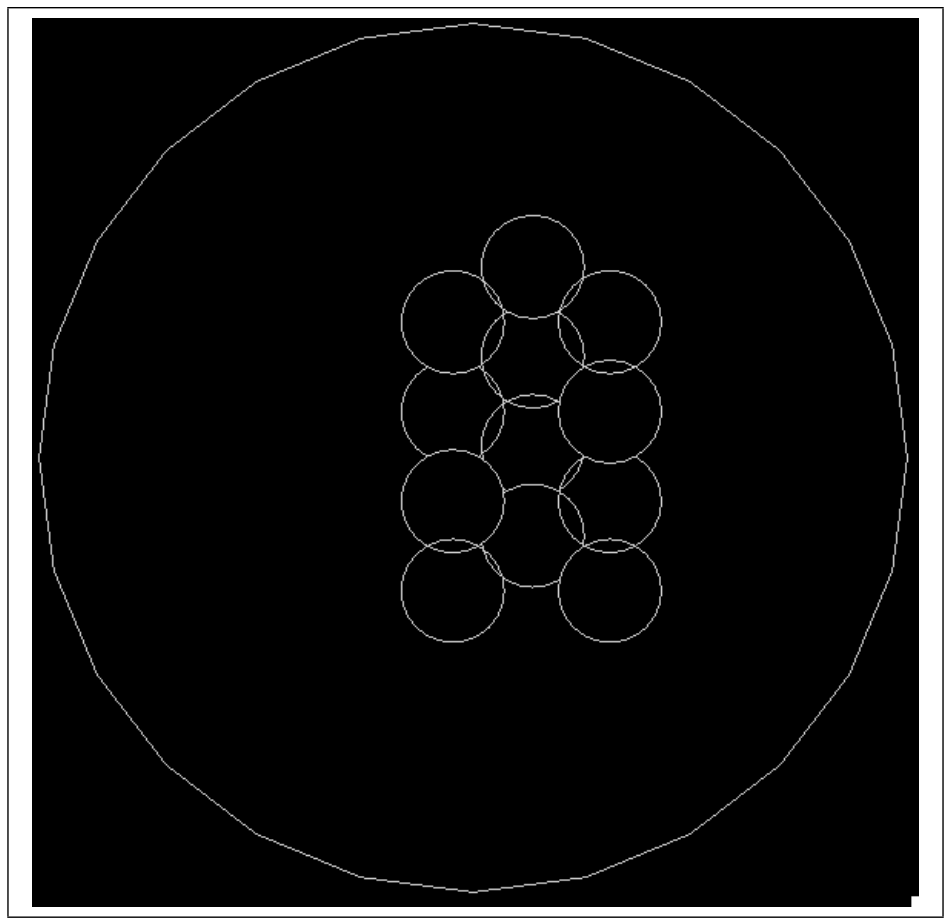

Figure 4: Compensator modeled with subtraction solids

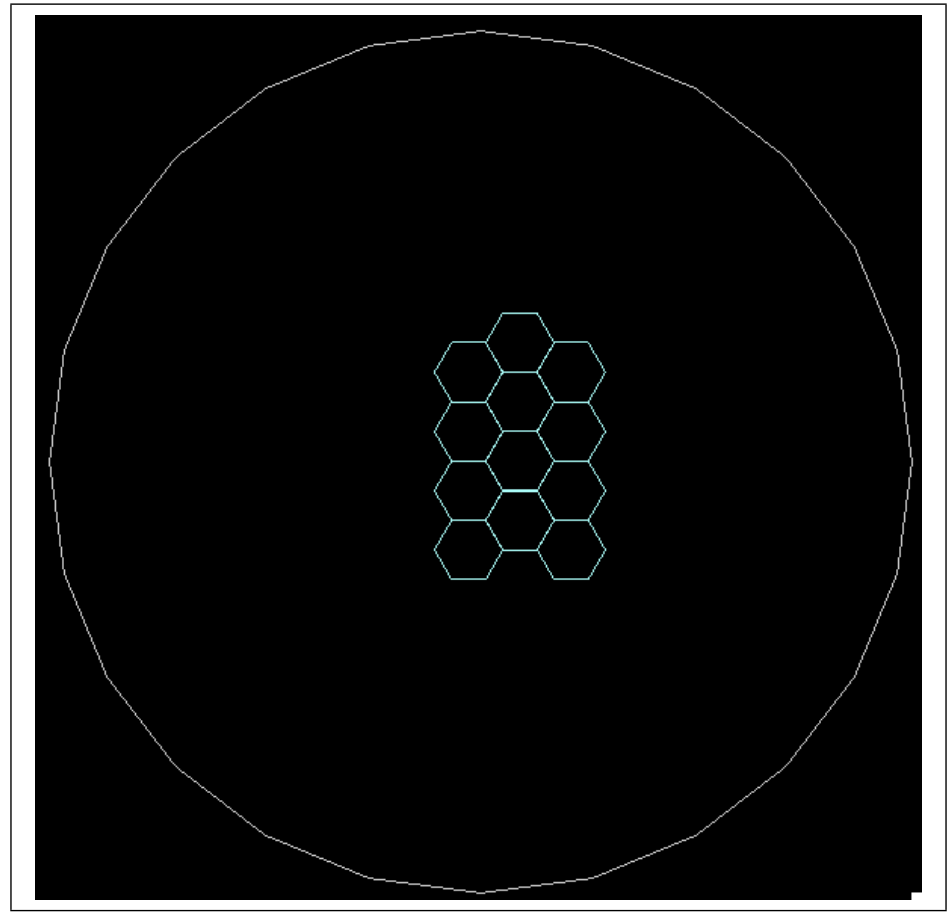

Figure 5: Compensator modeled with 3D hexagons 